\title{
Ultrasound Guided Saline Hydrostatic Reduction: A Non-Surgical Procedure for the Management of Intussusception in Children
}

\author{
Suvarna Latha1, P. M. Venkata Sai', R. K. Bagadi \\ ${ }^{1}$ Department of Radiology, Sri Ramachandra University, Porur, Chennai, India \\ ${ }^{2}$ Pediatric Surgery, Sri Ramachandra University, Porur, Chennai, India \\ Email: kiranlathap@yahoo.co.in
}

Received 3 April 2014; revised 13 May 2014; accepted 3 July 2014

Copyright (C) 2014 by authors and OALib.

This work is licensed under the Creative Commons Attribution International License (CC BY). http://creativecommons.org/licenses/by/4.0/

(c) (i) Open Access

\begin{abstract}
Introduction: Intussusception is the most common cause of intestinal obstruction in infant \& toddler age group. Intussusception is the telescoping of one segment of bowel into the contiguous distal segment. Peak age incidence is between 2 months to 3 years of life. Objective of the study: To prove the efficacy of Ultrasound guided saline hydrostatic reduction, a non surgical, non radiation method in the management of intussusception in children. Materials and methods: Fifty-three children in the age group of 2 months to 3 years with clinical diagnosis of intussusception on whom the Ultrasonogram (USG) guided hydrostatic reduction with normal saline were attempted. $500-1000 \mathrm{ml}$ of normal saline was used for reduction. High resolution USG (7.5 MHz) was used to assess the progress of reduction during the procedure. Results: Our initial experience in the last six years from April 2007 to March 2013 showed successful reduction in 45 children out of 53 attempted reductions.
\end{abstract}

\section{Keywords}

Intussusception, Hydrostatic Reduction, Ultrasonography (USG)

Subject Areas: Clinical Trials, Pediatrics

\section{Introduction}

Intussusception is one of the common causes of intestinal obstruction in children with peak incidence between 2 months to 3 years of age [1]. Intussusception is invagination of a segment of proximal bowel into the contiguous

How to cite this paper: Latha, S., Venkata Sai, P.M. and Bagadi, R.K. (2014) Ultrasound Guided Saline Hydrostatic Reduction: A Non-Surgical Procedure for the Management of Intussusception in Children. Open Access Library Journal, 1: e644. http://dx.doi.org/10.4236/oalib.1100644 
lumen of distal bowel loop. The proximal telescoping loop is called as intussusceptum and the distal loop is called as intussuscipiens [1]. When this happens, the mesentery and blood vessels, which have telescoped along with the intussusceptum, will get compressed in the outer loop resulting in ischemia and edema of the bowel wall presenting with severe pain.

If intussusception is not diagnosed and not relieved, it may result in complications like bowel wall necrosis and perforation.

Fifty percent are affected in the first year of life [1] and $24 \%$ presenting in the second year. Approximately 2/3 of patients are boys. Most common cause of intussusception in this age group is idiopathic.

If intussusception occurs outside the above mentioned age group, possibility of pathologic lead point is to be suspected as a cause of intussusceptions (Figure 1).

\section{Symptoms}

In our study, 35 children presented with abdominal pain and palpable abdominal mass. Eight children presented with features of intestinal obstruction, another ten children presented with a combination of abdominal pain and bloody diarrhea.

\section{Materials and Methods}

The study includes the patients for a period of 6 years, from April 2007 to March 2013.

A total number of 53 cases clinically diagnosed as intussusception and confirmed by ultrasonographic appearance using a linear array transducer of 7.5 - $10 \mathrm{MHz}$ using ALOKA SSD 5500 Ultrasound machine.

Patients were evaluated further for viability of the bowel by demonstrating the vascularity using color Doppler.

The following criteria were used to decide the suitability of the patient for the Ultrasound guided saline hydrostatic reduction.

\subsection{Inclusion Criteria}

1) Patients presenting within 48 hours of onset of symptoms.

2) Presence of palpable mass for more than 6 hours.

3) Absence of signs of perforation and peritonitis, including no radiographic evidence of free intraperitoneal air (especially in whom large amount of free fluid was seen in the peritoneum).

\subsection{Exclusion Criteria}

Absolute contra indications are perforation, peritonitis, severe dehydration and hypovolemic shock.

Other contraindications include:

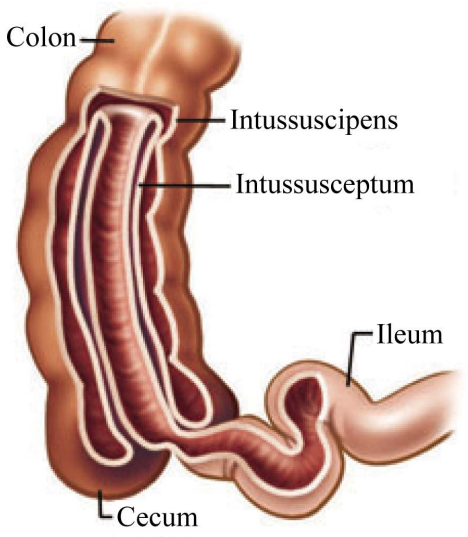

Figure 1. Intussusception is telescoping of one loop of bowel in to the other. Herniating loop is called intussusceptum and the distal loop is called Intussuscipiens. 
- Absence of vascularity on color Doppler imaging;

- Appearance of "Dissection sign";

- Presence of large amount of free fluid in the peritoneal cavity.

\subsection{Procedure}

The patient's parents were explained about the procedure in detail and informed consent was obtained.

The pediatric surgical team was also present in ultrasound room throughout the procedure.

The child was well hydrated before the procedure, for the best results [1].

A 16 F Foley's catheter introduced into the rectum with the child put on left lateral position, after lubricating tip of the catheter with $2 \%$ Lignocaine.

The Foley's bulb was then inflated with about $10-15 \mathrm{ml}$ of normal saline. The buttocks taped together tightly to provide a seal. The other end of the catheter was connected to a bottle of normal saline (at normal body temperature) through an intravenous line and the saline bag was suspended at more than $100 \mathrm{~cm}$ above the bed level and normal saline was allowed to flow into the rectum under gentle hydrostatic pressure.

Distending colonic loops and gradual passage of saline into the ileal loops monitored under real time Ultrasonography [1]-[3]. Complete reduction was assumed once the passage of saline through the ileo-cecal valve into the terminal ileum was seen (Figure 3). Saline flow was allowed into several loops of ileum. $500-1000 \mathrm{ml}$ of saline was used depending up on the size of the patient. The amount of saline required was recorded in each case.

Once reduction was found to be achieved, the saline was drained by lowering the saline bag below the level of the bed. Following this, Foley's bulb was deflated and the catheter was removed. Clinical condition of the patient was closely and carefully monitored throughout the procedure (Figure 2 and Figure 3).

If the intussusception is not reduced after a 3 minutes of sustained pressure, the saline pressure has to be lowered and child should be rested for 3 min. Three such attempts can be made before considering the intussusception irreducible and sending the patient for surgical management.

Procedure should be abandoned if the saline dissects between the layers of intussusception. This sign is described as "dissection sign". Which indicates that the "reduction is less likely" to happen by nonsurgical method. [4].

After the procedure the patient was shifted to observation room under pediatrician's supervision. A course of antibiotic therapy was started. In each case follow-up, High Resolution Ultrasound was done after 24 hours to rule out recurrence [5].

\section{Results}

Forty-five cases out of fifty-three were successfully reduced using saline hydrostatic reduction under ultrasonography guidance. In seven children in whom, the Ultrasonogram (USG) showed an appearance of ileoileocolic

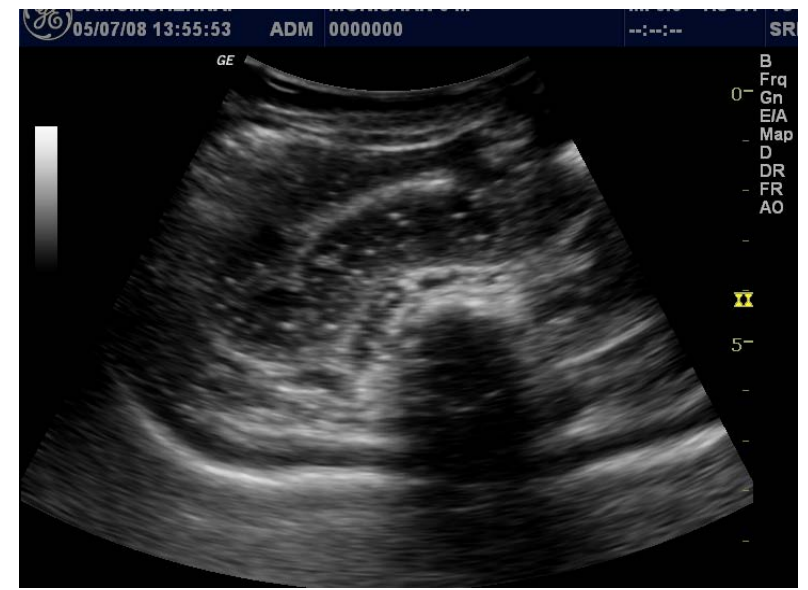

Figure 2. Intussusception: Ultrasound image of the colon during the procedure. Colonic loop distending with gradual passage of saline. 


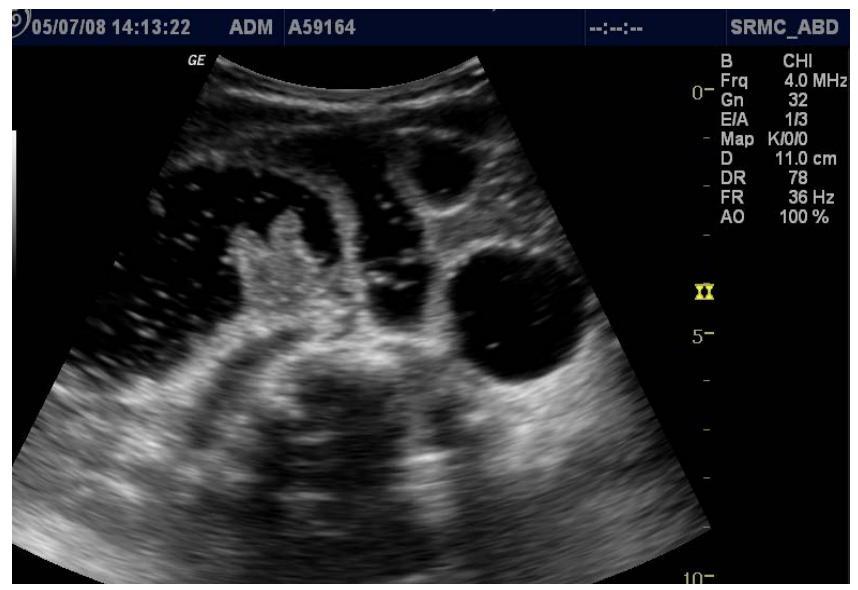

Figure 3. Intussusception: Ultrasound image during the procedure. Saline is distending the ileo-caecal region and outlining the ileocaecal valve. Complete reduction was assumed once the passage of saline through the ileo-caecal valve into the terminal ileum was seen.

intussusception (double loop intussusception), ultrasound guided reduction was attempted in view of positive inclusion criteria. Real time monitoring showed accumulation of Saline between the loops of intussusceptum and intussuscipiens. No saline entered into the ileal loops even after introducing around $500 \mathrm{ml}$, at 3 safe successive attempts of 3 minutes each suggesting the need for surgical management. Procedure abandoned and the reduction was achieved surgically.

Bowel perforation occurred in one patient, which was eventually managed surgically.

\section{Discussion}

There are several types of intussusception described depending upon the anatomical parts of the bowel involved, such as (a) Ileo Colic, (b) Ileo Ileal, (c) Colo Colic, (d) Ileo Ileo Colic, (e) multiple and (f) retrograde.

The Ileo Colic types of Intussusceptions are the commonest type constituting about $90 \%$ of cases [1].

\subsection{Incidence}

Majority of Intussusceptions are idiopathic. 50\% occur in first year and two-thirds of the patients are boys.

Outside this age group there is an increased incidence of lead points e.g. polyps, meckel's diverticulum, nonHodgkin's lymphoma, duplication cyst.

\subsection{Clinical Presentation}

1) Intermittent colicky abdominal pain is the most common presentation;

2) Palpable abdominal mass;

3) Intestinal obstruction;

4) Vomiting;

5) Diarrhea, sometimes with blood in the stool;

6) Some patients present with dehydration, hypovolemic shock and lethargy intermittent.

\subsection{Imaging Evaluation}

Plain X-ray abdomen may show a soft tissue mass and signs of intestinal obstruction, sometimes free air.

Presence of free air suggests perforation which is a contraindication for hydrostatic reduction.

Ultrasound on transverse scan may show, a "doughnut sign", central echogenic mucosa with surrounding edematous wall of the intussusceptum or a "target sign", concentric rings of alternating sonolucency and echogenicity. On longitudinal scan a "pseudo kidney sign" is evident. 
These characteristic signs are easily recognized at the leading edge of intussusceptions (Figure 4 and Figure 5).

Presence of large amount of free fluid in the peritoneal cavity is a contraindication where as small amount is common in intussusception and does not contraindicate the procedure.

Color Doppler can detect the viability of involved bowel by showing presence or absence of flow.

Absence of blood flow in color Doppler study in the intussusception suggests bowel ischemia and correlates significantly with irreducibility.

\subsection{Treatment Options Available Are}

- Operative reduction

- Non operative reduction using procedures like:

a) USG guided reduction using normal saline;

b) Fluoroscopy guided air enema reduction.

USG guided reduction using normal saline is the recently adopted technique. This method uses about 500 $1000 \mathrm{ml}$ of normal saline at a height of $100-130 \mathrm{~cm}$, maintaining a constant hydrostatic pressure [1]-[7].

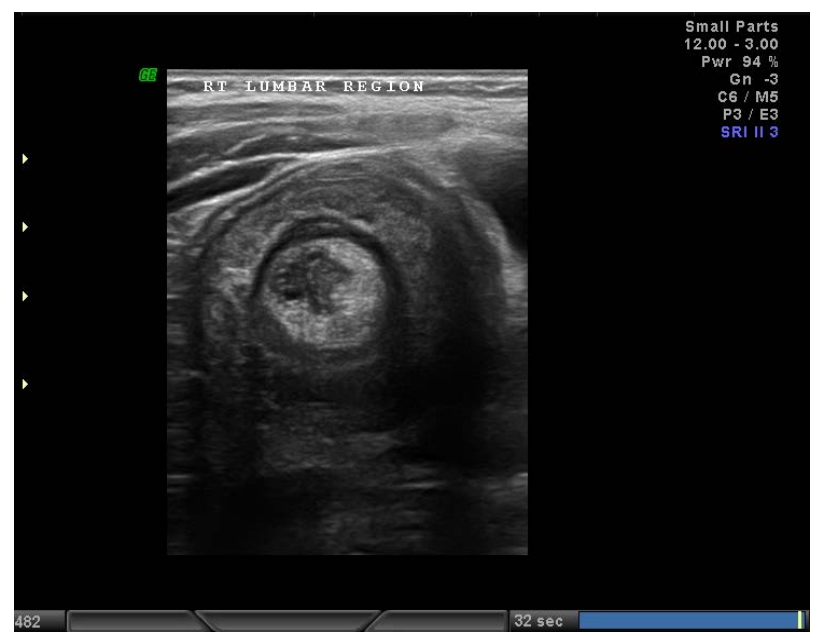

Figure 4. Intussusception: Doughnut sign or Target sign. Ultrasound appearance of concentric layers of alternating echogenic and hypoechogenic bands.

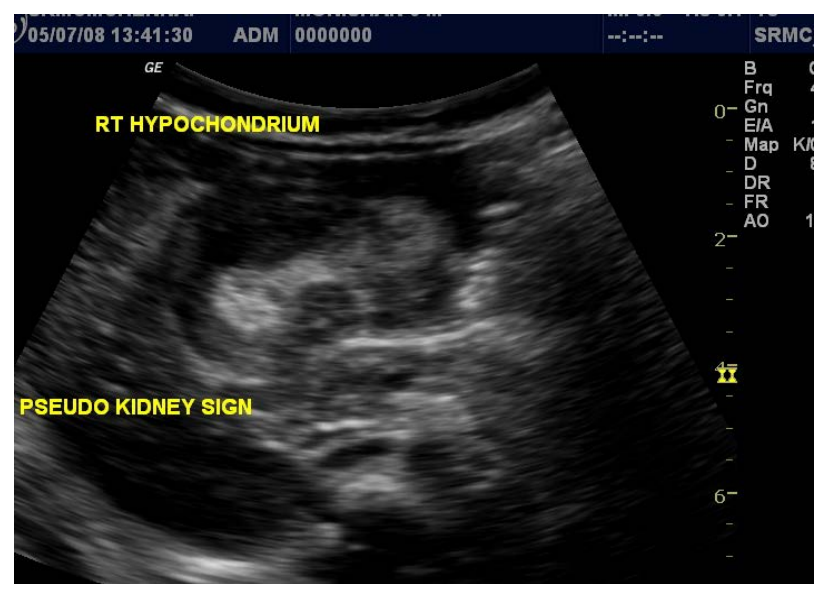

Figure 5. Intussusception: Pseudo kidney sign. Ultrasound appearance of oblique longitudinal section of the intussuscepted bowel. Echogenic mesentery herniated along the Intussuscipiens appears like hilum of the kidney. 


\subsection{Non Operative Reduction Is Not Recommended in the Following}

- Absence of vascularity on color Doppler imaging;

- Appearance of “dissection sign”;

- Presence of large amount of free fluid in the peritoneal cavity;

- No signs of reduction even after three attempts of three minutes each with an interval rest period of 3 minutes [7].

However our experience showed that in the presence of pediatric surgery team readily available in the procedure room to deal with any complications if they arise and if the patient is able to tolerate the procedure, the number of attempts can be increased up to 5 and the duration of each attempt can be prolonged up to 5 minutes.

\subsection{Advantages}

1) The procedure is simple, easily available, effective, and economical;

2) Facilitates Real time monitoring. This helps to observe and confirm complete reduction.

Also allows immediate identification of perforation in case it happens.

3) Pre-procedural ultrasound and color Doppler assessment helps improving the percentage of positive outcome of non surgical management;

4) No radiation hazard;

5) Less morbidity;

6) Real time observation under ultrasound avoids the pitfall of pseudo-reduction as seen in fluoroscopic guided procedures;

7) Disadvantages of air insufflation like fluctuation of intra colonic pressure can be avoided.

Disadvantages of barium enema (messy procedure \& Barium peritonitis, if perforation occurs) are overcome by saline reduction.

8) Recurrence can also be treated by the same method;

9) Can be readily repeated in case of recurrent intussusception.

\subsection{Limitations}

1) The person performing the procedure needs to have expertise to perform real time ultrasound scan on a pediatric patient;

2) The child usually struggles during the saline infusion due to discomfort, making the real time ultrasound more difficult;

3) Risk of perforation is high if the Foley's balloon is inflated;

4) Saline leaks though the anal canal if the Foley's balloon is not inflated and adequate pressure will not be built to reduce the intussusceptions.

\section{Conclusions}

No more complications! No more radiation hazard! No more mess!

USG-guided hydrostatic reduction of intussusception in children using normal saline is a simple and cost effective technique which requires minimal hospital stay.

USG guidance equipped with color Doppler helps proper patient selection which improves the success rate of this non surgical managemen.

\section{References}

[1] Applegate, K.E. Intussusception, Chapter 108, Caffey’s Pediatric Diagnostic Imaging. 12th Edition.

[2] Bolia, A.A. (1985) Diagnosis and Hydrostatic Reduction off an Intussusception under Ultrasound Guidance-Case Report. Clinical Radiology, 36, 655-657. http://dx.doi.org/10.1016/S0009-9260(85)80269-5

[3] Peh, W.C., Khong, P.L. and Chan, K.L. (1996) Sonographically Guided Hydrostatic Reduction of Childhood Intussusception Using Hartmann's Solution. American Journal of Roentgenology, 167, 1237-1241. http://dx.doi.org/10.2214/ajr.167.5.8911188

[4] Fishman, M.C., Borden, S. and Cooper, A. (1984) The Dissection Sign of Nonreducible Ileocolic Intussusception. 
American Journal of Roentgenology (AJR), 143, 5-8. http://www.ncbi.nlm.nih.gov/pubmed/6610330

[5] Kumar, K., Hameed, S. and Umamaheswari (2006) Ultrasound Guided Hydrostatic Reduction in the Management of Intussusception. The Indian Journal of Paediatrics, 73, 217-220.

[6] Khong, P.L., Peh, W.C. and Lam, C.H. (2000) Ultrasound-Guided Hydrostatic Reduction of Childhood Intussusception: Technique and Demonstration. Radiographics, 20, 1818.

[7] Manesh, Y., Glover-Addy, H. and Twire, V.E. (2011) Ultrasound Guided Hydrostatic Reduction of Intussusception in Children at Korle Bu Teaching Hospital, an Initial Experience. Ghana Medical Journal, 45, 128-131. 